\section{OP0162-HPR ESTIMATING HEALTH-RELATED QUALITY OF LIFE FOR GOUT PATIENTS: A POST-HOC ANALYSIS OF UTILITIES FROM THE CLEAR TRIALS}

F. Pérez-Ruiz ${ }^{1}$, A. So ${ }^{2}$, P. Kandaswamy ${ }^{3}$, R. Karra ${ }^{3}$, K. Kelton ${ }^{4}$, S. Perk ${ }^{4}$, T. Bardin ${ }^{5} .{ }^{1}$ University of the Basque Country, Biscay, Spain; ${ }^{2}$ Service de rhumatologie, Université de Lausanne, Lausanne, Switzerland; ${ }^{3}$ Grünenthal $\mathrm{GmbH}$, Aachen, Germany; ${ }^{4}$ Medical Decision Modeling, Inc., Indianapolis, USA; ${ }^{5}$ Rhumatologie, Lariboisière Hospital, and Université Paris Diderot Sorbonne Cité, Paris, France

Background: Prior studies of health-related quality of life (HRQoL) in gout patients have shown significant disutilities associated with flares and tophi or serum uric acid (sUA). ${ }^{12}$ However, no prior study has explored the simultaneous impact of all three aspects of gout severity.

Objectives: Estimate the impact of gout flares, tophi, and sUA levels on HRQoL through post-hoc analysis of SF-36 data collected in two randomised, doubleblind, placebo-controlled Phase 3 studies of urate lowering therapies in gout patients (CLEAR 1 and CLEAR 2). ${ }^{34}$

Methods: Linear regression analysis was used to estimate the effects of patient and disease characteristics on SF-6D scores at month 6 and month 12. Multiple regression methods and out-of-sample testing were used to select the final model from covariates representing tophus burden, the number of flares during each sixmonth period, serum urate levels, baseline characteristics, and comorbidities. Predicted mean utility scores were calculated by evaluating the model at the mean values of the covariates (excluding location-specific covariates) in the pooled CLEAR 1 and CLEAR 2 intent-to-treat population. ${ }^{34}$

Results: The final regression model (table 1) includes significant disutilities associated with the presence of tophi at screening $(0.0418$; SE: $0.0073 ; p<0.001)$, the number of gout flares $(0.0036$ per flare; SE: $0.0005 ; p<0.001)$, and median sUA on-treatment $(0.0083$ per $\mathrm{mg} / \mathrm{dL}$ in excess of $6 \mathrm{mg} / \mathrm{dL}$; SE: $0.0031 ; p=0.007)$. The predicted mean utility scores for patients with zero flares and no tophi are 0.7718 for sUA $<6 \mathrm{mg} / \mathrm{dL} ; 0.7552$ for sUA 6 to $<8 \mathrm{mg} / \mathrm{dL} ; 0.7386$ for sUA 8 to $<10 \mathrm{mg} / \mathrm{dL}$; and 0.7220 for $\mathrm{SUA} \geq 10 \mathrm{mg} / \mathrm{dL}$.

Abstract OP0162HPR - Table 1. Regression model of SF-6D utilities

\begin{tabular}{|c|c|c|c|}
\hline Coefficient & Mean & SE & $\begin{array}{c}\mathbf{p} \\
\text { value }\end{array}$ \\
\hline Intercept & 0.9182 & 0.0235 & $<0.001$ \\
\hline Region Europe & $\begin{array}{c}- \\
0.0641\end{array}$ & 0.0092 & $<0.001$ \\
\hline Country New Zealand & 0.0523 & 0.0200 & 0.009 \\
\hline $\operatorname{Age}^{\dagger}$ & $\begin{array}{c}- \\
0.0009\end{array}$ & 0.0003 & 0.003 \\
\hline Female & $\begin{array}{c}- \\
0.0242\end{array}$ & 0.0140 & 0.084 \\
\hline Black race & $\begin{array}{c}- \\
0.0307\end{array}$ & 0.0098 & 0.002 \\
\hline Body mass index ${ }^{\dagger}$ & $\begin{array}{c}- \\
0.0020\end{array}$ & 0.0005 & $<0.001$ \\
\hline Diabetes mellitus ${ }^{\dagger}$ & $\begin{array}{c}- \\
0.0215\end{array}$ & 0.0080 & 0.007 \\
\hline Hypertension ${ }^{\dagger}$ & $\begin{array}{c}- \\
0.0172\end{array}$ & 0.0067 & 0.010 \\
\hline Unemployed ${ }^{\dagger}$ & $\begin{array}{c}- \\
0.0596\end{array}$ & 0.0103 & $<0.001$ \\
\hline Disabled or retired due to illness ${ }^{\dagger}$ & $\begin{array}{c}- \\
0.1125\end{array}$ & 0.0121 & $<0.001$ \\
\hline Number of gout flares & $\begin{array}{c}- \\
0.0036\end{array}$ & 0.0005 & $<0.001$ \\
\hline Tophi present at screening & $\begin{array}{c}- \\
0.0418\end{array}$ & 0.0073 & $<0.001$ \\
\hline $\mathrm{sUA}^{\star}$ per $\mathrm{mg} / \mathrm{dL}$ in excess of $6 \mathrm{mg} / \mathrm{dL}$ & $\begin{array}{c}- \\
0.0083\end{array}$ & 0.0031 & 0.007 \\
\hline
\end{tabular}

$R^{2}$ : 0.1788; Adjusted $R^{2}$ : 0.1730; Residual standard error: 0.1217 on 1857 degrees of freedom. "Baseline characteristics. "Median sUA on-treatment. Abbreviations: SE, standard error; SF-6D, Short form 6D questionnaire; SUA, serum uric acid.

Conclusions: The present analysis is unique in that it explored the simultaneous effects of flares, tophi, and SUA on HRQoL. The results indicate that high sUA levels are associated with significant disutility when controlling for the effects of flares, tophi, and other patient covariates. The clinical rationale is that uncontrolled sUA creates a state of chronic inflammation, contributing to underlying ill health in addition to its effects on flares, tophi, and comorbidities.
REFERENCES:

[1] Beard SM, et al. Eur J Health Econ 2014;15(5):453-63.

[2] Khanna PP, et al. Health Qual Life Outcomes 2012;10:117.

[3] Data on file. Clinical Study Report RDEA594-301. Ardea Biosciences, Inc.; 2014.

[4] Data on file. Clinical Study Report RDEA594-302. Ardea Biosciences, Inc.; 2014.

Disclosure of Interest: F. Pérez-Ruiz Grant/research support from: Spanish Society of Rheumatology, Cruces Hospital Rheumatology Association, Consultant for: Grünenthal, Menarini, Speakers bureau: Grünenthal, Menarini, A. So Consultant for: Grünenthal, Sobi, Speakers bureau: Grünenthal, Sobi, P. Kandaswamy Employee of: Grünenthal, R. Karra Employee of: Grünenthal, K. Kelton Employee of: Medical Decision Modelling, a contractor to Grünenthal, S. Perk Employee of: Medical Decision Modelling, a contractor to Grünenthal, T. Bardin Consultant for: Grünenthal, AstraZenaca, Menarini, Ipsen, Sobi, Novartis DOI: 10.1136/annrheumdis-2018-eular.6822

\section{OP0163-HPR DEVELOPMENT OF AN ONLINE SELF-MANAGEMENT PLATFORM FOR PEOPLE WITH RHEUMATIC AND MUSCULOSKELETAL CONDITIONS (MSKHUB.COM)}

Y Prior $^{1,2}$, L. Sammut ${ }^{1,3}$, C. Vasilica ${ }^{1}$, on behalf of the MSKHUB Patient and Public Research Advisory Group (PRAG) and the MSKHUB Health Professionals Reference Group (HPRG). ${ }^{1}$ Health Sciences, University of Salford, Salford; ${ }^{2}$ Rheumatology, MidCheshire Hospitals NHS Trust, Leighton Hospital, Crewe; ${ }^{3}$ Rheumatology, University Hospital Southampton NHS Foundation Trust, Southampton, UK

Background: Patient information and education have been shown to improve pain and self-efficacy and increase overall quality of life in people with chronic musculoskeletal conditions (MSCs). Informed patients are better able to distinguish and manage symptoms, use treatments effectively, access services needed, manage work and cope better with the psychological impact of their conditions. However, there is a need to improve the access to high quality specialist health information for people with rheumatic and MSCs.

Objectives: This study aimed to test the usability and acceptability of an online self-management platform developed for people with rheumatic and MSCs to facilitate access to (i) valid and reliable health information (ii) evidence-based Patient Reported Outcome Measures (PROMs) (iii) advice on self-help, assistive technologies and rehabilitation (iv) and peer support via an online community.

Methods: Phase 1 involved the MSKHUB community development through service-user and health professionals group formation. People with Rheumatic and MSCs $(n=15)$ and their family/carers $(n=5)$ were purposively identified via social media networks and brought in as collaborators to form the MSKHUB Patient and Public Research Advisory Group (PRAG). A multi-disciplinary group of health professionals $(n=7)$ were also identified through the academic and clinical networks to form the MSKHUB Health Professionals Reference Group (HPRG). A digitallead specialising in health technologies and social media networks also supported the development of the online content and social networks. The digital lead PRAG and HPRG had face-to-face and online meetings throughout the Phase 1 to inform the development of the MSKHUB through an iterative process. Following the Phase 1, 25 participants with rheumatic and MSCs used the website on their own devices at home and provided feedback by completing a user-experience survey assessing ease of use, helpfulness, and satisfaction with the MSKHUB.

Results: Participants completed the registration process and all PROMs on the MSKHUB site in an average of 1.5 hours (SD $30 \mathrm{~min}$ ); some PROMs required more to complete due to their size and complexity but all PROMs were deemed as relevant and important to include. Participants' comments were addressed to improve the user-friendliness and ease of use. Participants reported high levels of usefulness and satisfaction in the user-experience survey $(80 \%$ rated the usefulness as $9 / 10 \%$ and $92 \%$ rated satisfaction as $7 / 10$ [1=not useful/not satisfied at all to $10=$ extremely useful/satisfied]). All participants indicated they would continue using the MSKHUB and recommend it to their families and/or friends with rheumatic and MSCs.

Conclusions: MSKHUB Development (Phase 1) involved the creation and testing the usability and acceptibility of an online self-management platform for people with rheumatic and MSCs, which is an important step in developing appropriate and user-friendly online health education platforms. Our study suggested that community dwelling people with rheumatic and MSCs demonstrated a high degree of acceptance of the MSKHUB and could use it easily. The Phase 2 will involve a wider testing of the MSKHUB for general site activity, use and evaluation through a variety of embedded appraisal methods within the sit is currently underway. 
Acknowledgements: This study was funded by the Logres Trust.

Disclosure of Interest: None declared

DOI: 10.1136/annrheumdis-2018-eular.7164

\section{OP0164-HPR IMPACT OF A NURSE-LED PROGRAM OF PATIENT SELF-ASSESSMENT AND SELF-MANAGEMENT AXIAL SPONDYLOARTHRITIS: RESULTS OF A PROSPECTIVE, MULTICENTRE, RANDOMISED, CONTROLLED TRIAL (COMEDSPA)}

A. Moltó $^{1}$, A. Etchieto ${ }^{1}$, S. Poiraudeau ${ }^{1}$, L. Gossec ${ }^{2}$, P. Claudepierre ${ }^{3}$,

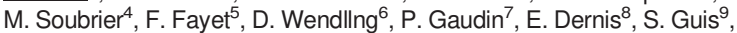
S. Pouplin ${ }^{10}$, A. Ruyssen-Witrand ${ }^{11}$, M. Dougados ${ }^{1}$. ${ }^{1}$ Cochin; ${ }^{2}$ Pitié-Salpêtrière; ${ }^{3}$ Henri Mondor, Paris; ${ }^{4} \mathrm{CHU}$ Clermont-Ferrand, Clermont-Ferrand; ${ }^{5} \mathrm{CHU}$ Clermont-Ferrand, Paris; ${ }^{6} \mathrm{CHU}$ Besançon, Besançon; ${ }^{7} \mathrm{CHU}$ Grenoble, Grenoble; ${ }^{8} \mathrm{CH}$ Le Mans, Le Mans; ${ }^{9} \mathrm{AP}-\mathrm{HM}$, Marseille; ${ }^{10} \mathrm{CHU}$ Rouen, Rouen; ${ }^{11} \mathrm{CHU}$ Toulouse, Toulouse, France

Background: Nurses should promote self-assessment and self-management skills in order that patients might achieve a greater self efficacy and improvement in patients with axSpA.

Objectives: To evaluate the impact of a nurse-led program of self-management/assessment for disease activity program in axSpA.

Methods: Prospective, randomised, controlled, open, 12 month trial (NCT02374749). Participants 1/Patients: consecutive Axial SpA patients (according to rheumatologist) attending a clinic of the participating centres were invited $0.2 /$ Nurses :all participated at a 1 day meeting prior the start of the study. Study treatment: a program including: 1) Self-management=a) a video explaining the disease, the interest of smoking cessation in axial SpA, the role of NSAIDs as cornerstone treatment in axSpA in the absence of contra-indications, the interest of physical activity and exercise, followed by a discussion with the nurse; $b$ ) physical examination by the nurse to check for the presence of spinal deformitiesand depending on the absence/presence of such deformities projection of a specific video of home-based exercises. 2) Self-assessment: Video presentation of the rationale of the use of a composite index (ASDAS/BASDAI), followed by discussion with the nurse. Explanation by the nurse of the collection, calculation of BASDAI and ASDAS Treatment allocation: after written informed consent, the treatment was allocated randomly via en electronic system. Outcome variables: Primary: The level of coping ( $0-10$, where $0=$ very well) after 12 months. Other variables: Successful smoking cessation, NSAID intake, Number of home-based or supervised exercise, international physical activity questionnaire (IPAQ)

Results: Baseline characteristics of the 502 recruited patients (250 and 252 in the active and control groups, respectively): Age: $46.7 \pm 12.2$ years, male gender: $62.7 \%$, disease duration: $13.7 \pm 11.0 \mathrm{y}$, Xray sacroiliitis $62.8 \%$, MRI sacroiliitis $65.7 \%$, current biologic treatment: $78.3 \%$, ASDAS-CRP: $1.9 \pm 0.8$, BASFI: 25.6 \pm 22 .3. After 1 year, coping levels were lower in the active group, but not significant $(2.8 \pm 2.0$ vs $3.0 \pm 2.1, p=0.3)$. However, there was a significant decrease in the BASDAI in the active group (- $1.2 \pm 15.8 \mathrm{vs}+1.4 \pm 15.7, \mathrm{p}=0.03)$, a significant increase in the number $(6.1 \pm 28.8$ vs $-0.4 \pm 26.9, \mathrm{p}=0.03)$ and duration (4.3 \pm 20.1 vs $-1.7 \pm 20.7, p<0.01$ ) of the home-exercises in the active group, and a greater IPAQ score in the active group at the end of follow-up (138.4 \pm 227 vs 95.6 $\pm 173, \mathrm{p}=0.02$ ).

Conclusions: This study highly suggests a short-term benefit of a nurse led program on the self-management and self-assessment for disease activity in a young axSpA population in particular with regard to the frequency and the duration of home exercises.

Acknowledgements: This study was conducted thanks to a grant from the French National Research Program (PHRC) thanks to an unrestricted grant from ABBVIE.

Disclosure of Interest: None declared

DOI: 10.1136/annrheumdis-2018-eular.4181

\section{THURSDAY, 14 JUNE 2018}

\section{Fires and firefighters: switching the immune system on and off}

\section{OP0165 \\ JOINT-SPECIFIC DIFFERENCES IN THE ACTIVATION OF THE JAK-STAT PATHWAY IN RHEUMATOID ARTHRITIS}

T. Masterson, K. Klein, E. Karouzakis, O. Distler, C. Ospelt, M. Frank Bertoncelj. Center of Experimental Rheumatology Zurich, University Hospital Zurich, Schlieren, Switzerland

Background: Synovial fibroblasts (SF) promote chronic joint inflammation and joint destruction in rheumatoid arthritis (RA). We have shown recently that SF from different joints exhibit profound differences in their transcriptomes, epigenomes and functions, which creates a unique microenvironment in each joint. This might influence the susceptibility of distinct joints to develop RA or lead to joint-specific differences in the disease severity or therapeutic response.

Objectives: To analyse differences in the JAK-STAT pathway in SF from different joints.

Methods: SF were isolated from knee, shoulder and hand joints of RA and osteoarthritis patients undergoing joint replacement surgery and from knee synovia biopsies of non-arthritic subjects with arthralgia. Transcriptomes and epigenomes of SF were determined by RNA-seq, Illumina HiSeq $2000 n=21$ ), ChIP-seq (Illumina HiSeq 2500, $n=7)$ and Infinium HumanMethylation450 BeadChip $(n=12)$. We used MetaCore (Thomson Reuters) for the pathway enrichment analysis of RNA-seq data. SF were stimulated with IL-6/soluble IL-6 receptor (IL-6/sIL-6R, $50 \mathrm{ng} / \mathrm{ml}$ each). The amount of STATs and phospho-STAT3 (p-STAT3) was measured by Western blot with normalisation to $\alpha$-tubulin.

Results: The JAK-STAT pathway was enriched in knee SF versus hand and shoulder SF (FDR<0.05). JAK1 (normalised reads - mean \pm SD: 10673 \pm 2084 ) and STAT1 (15520 \pm 2678$)$ were the top expressed Janus kinase and STAT mRNAs in SF, respectively, whereas the expression of JAK3 $(40 \pm 26)$ and STAT4

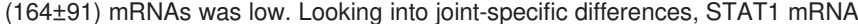
was higher in knee SF and shoulder SF compared with hand $S F(p<0.05$, FDR $<0.15$ ). Accordingly, STAT1 protein was increased in knee SF (STAT1/ $\alpha$ tubulin ratio: $0.83 \pm 0.02, p=0.02, n=4)$ and shoulder $S F(1.02 \pm 0.02, p=0.001, n=5)$ versus hand SF $(0.57 \pm 0.02, \mathrm{n}=3)$. JAK1, STAT2 and STAT5B mRNAs were higher in knee compared with hand SF $(\mathrm{p}<0.05$, FDR $<0.05)$ and STAT2 and STAT6 mRNAs were higher in knee versus shoulder $S F(p<0.05, F D R<0.05)$ TYK2 mRNA was high in hand SF compared with shoulder and knee SF $(p<0.05$, FDR $<0.05$ ). SF from different joints exhibited comparable DNA methylation at the promoters of these genes. Activating histone marks H3K4me3 and/or H3K27ac were enriched the promoters of JAK1, STAT1, STAT2 and STAT5B in knee versus hand SF. This indicated that the abundance of activating histone marks at gene promoters might shape joint-specific expression of a subset of Janus kinase and STAT genes. Stimulation of SF with IL-6/sIL-6R increased the phosphorylation of STAT3 in knee (p-STAT3/ $\alpha$-tubulin ratio $1.8 \pm 1.0, p=0.03, n=5)$ and shoulder SF $(1.8 \pm 0.7, p=0.03, n=6)$ compared with hand SF $(0.9 \pm 0.5, n=6)$. The basal amount of STAT3 protein and the ratio pSTAT3/STAT3 was higher in knee SF (STAT3/ $\alpha$-tubulin ratio: $0.8 \pm 0.1 ; p$-STAT3/STAT3: $2.2 \pm 0.8, n=3$ ) and shoulder SF $(0.6 \pm 0.4 ; 2.1 \pm 1, n=4)$ versus hand SF $(0.3 \pm 0.02 ; 1.0 \pm 0.4, n=2)$.

Conclusions: Here we show substantial quantitative and qualitative differences in the JAK-STAT signalling pathway in SF from different joints. Knee SF, in particular, exhibit increased expression of Janus kinase and STAT genes and enhanced JAK-STAT signalling upon stimulation with IL-6/sIL-6R. This suggests that RA in different joints might not be equally sensitive to Janus kinase inhibitors or blockade of IL-6. This has important implications in clinical practice and drug discovery in RA.

Disclosure of Interest: T. Masterson: None declared, K. Klein: None declared E. Karouzakis Grant/research support from: BTCure, GSK, O. Distler Grant/ research support from: Abbvie, Actelion, Bayer, Biogenldec, Boehringer Ingelheim, ChemomAb, espeRare foundation, Genentech/Roche, GSK, Inventiva iQone, Lilly, medac, Medlmmune, Mepha, MSD, Mitsubishi Tanabe Pharma, Novartis, Pfizer, Pharmacyclics, Sanofi, Sinoxa and UCB, Consultant for: Abbvie Actelion, Bayer, Biogenldec, Boehringer Ingelheim, ChemomAb, espeRare foundation, Genentech/Roche, GSK, Inventiva, iQone, Lilly, medac, Medlmmune Mepha, MSD, Mitsubishi Tanabe Pharma, Novartis, Pfizer, Pharmacyclics, Sanofi, Sinoxa and UCB, C. Ospelt Grant/research support from: euroTEAM BTCure, CABMM, IRR, Promedica, M. Frank Bertoncelj Grant/research support from: AbbVie Rheumatology grant 2017 euroTEAM, BTCure, IRR, Promedica, Georg und Berta Schwyzer Winiker Grant

DOI: 10.1136/annrheumdis-2018-eular.6708

\section{OP0166 COMPARATIVE EVALUATION OF CELLULAR AND MOLECULAR CHANGES ASSOCIATED WITH RESPONSE TO SELECTIVE IL-23 BLOCKADE VS DUAL IL-12/23 BLOCKADE IN PSORIASIS SKIN}

K. Li ${ }^{1}$, K. Campbell ${ }^{1}$, S. Garcet ${ }^{2}$, C. Brodmerkel ${ }^{1}$, J. Krueger ${ }^{2} .{ }^{1}$ Janssen Research and Development, LLC, Spring House; ${ }^{2}$ Laboratory for Investigative Dermatology, The Rockefeller University, New York, USA

Background: Emerging clinical data indicate that selective blockade of interleukin 23 (IL-23) can achieve greater efficacy compared to dual blockade of IL-12/23 in patients with moderate-to-severe psoriasis (PsO). ${ }^{1}$ Ustekinumab (UST) targets the p40 subunit common to IL-12 and IL-23, whereas guselkumab (GUS) specifically targets the IL-23-specific $\mathrm{p} 19$ subunit. While differences in antibody potency may explain therapeutic differences between UST and GUS, we explored cellula and molecular changes in the skin of PsO patients treated with UST or GUS to understand the mechanism underlying selective IL-23p19 inhibition. 